Network Working Group

Request for Comments: 1542

Updates : 951

Obsoletes: 1532

Category: Standards Track
W. Wimer

Carnegie Mellon University

October 1993

\title{
Clarifications and Extensions for the Bootstrap Protocol
}

Status of this Memo

This RFC specifies an Internet standards track protocol for the Internet community, and requests discussion and suggestions for improvements. Please refer to the current edition of the "Internet Official Protocol Standards" for the standardization state and status of this protocol. Distribution of this memo is unlimited.

Abstract

Some aspects of the BOOTP protocol were rather loosely defined in its original specification. In particular, only a general description was provided for the behavior of "BOOTP relay agents" (originally called BOOTP forwarding agents"). The client behavior description also suffered in certain ways. This memo attempts to clarify and strengthen the specification in these areas. Due to some errors introduced into RFC 1532 in the editorial process, this memo is reissued as RFC 1542 .

In addition, new issues have arisen since the original specification was written. This memo also attempts to address some of these.

Table of Contents

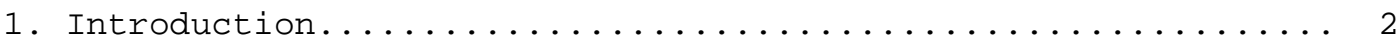

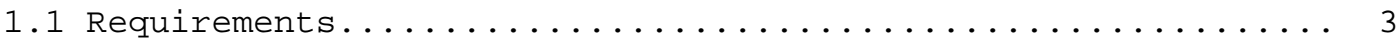

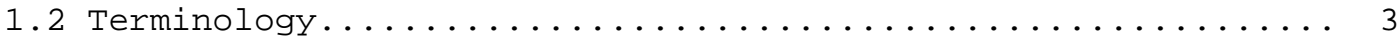

1.3 Data Transmission Order...................... 4

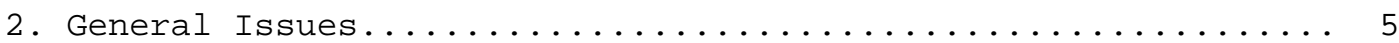

2.1 General BoOTP Processing...................... 5

2.2 Definition of the 'flags' Field..................... 5

2.3 Bit Ordering of Hardware Addresses................... 7

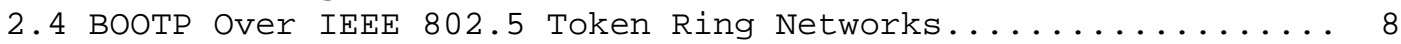

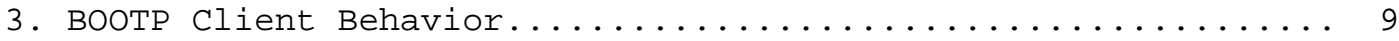

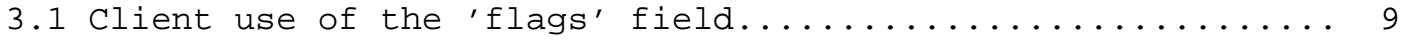



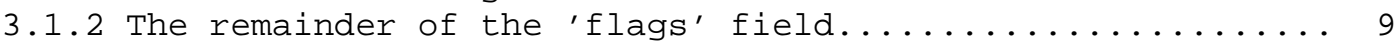

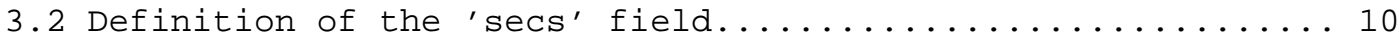

3.3 Use of the 'ciaddr' and 'yiaddr' fields................10 
3.4 Interpretation of the 'giaddr' field................ 11

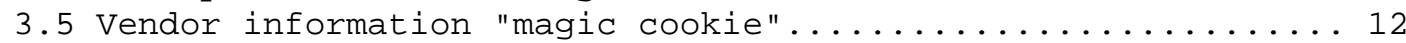

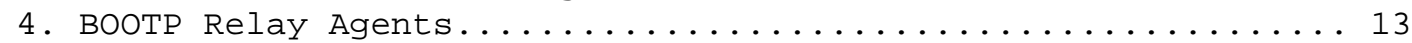

4.1 General BOOTP Processing for Relay Agents............... 14

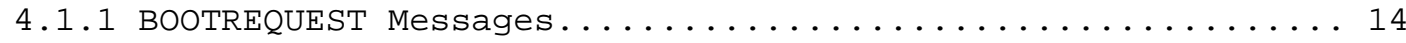

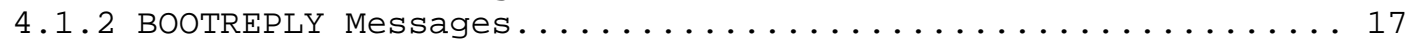

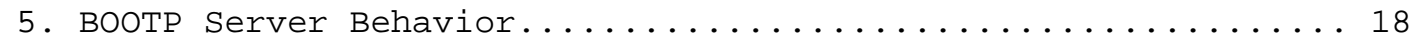

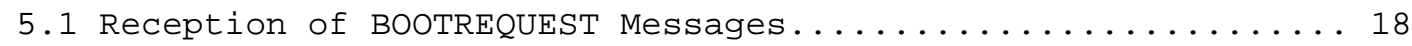

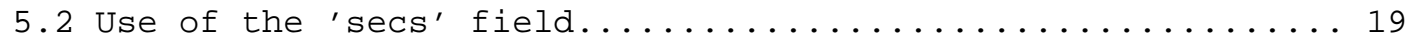

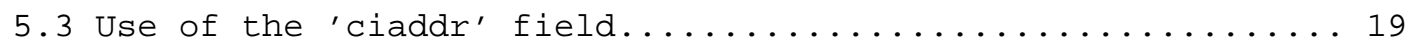

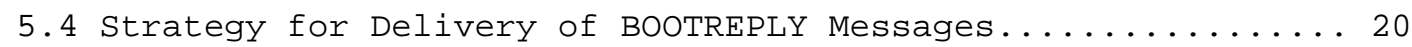

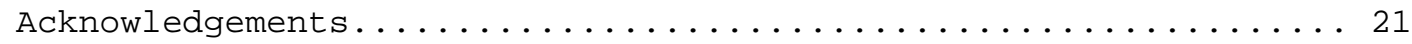

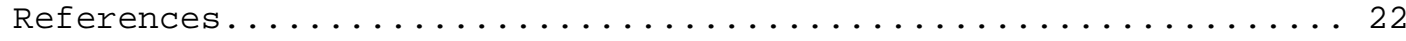

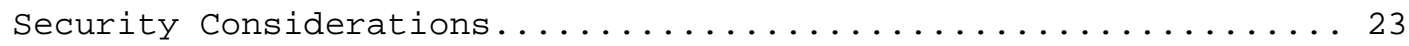

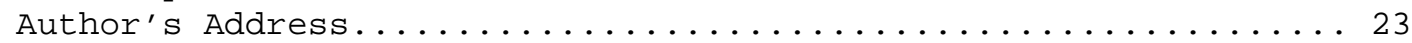

1. Introduction

The Bootstrap Protocol (BOOTP) is a UDP/IP-based protocol which allows a booting host to configure itself dynamically and without user supervision. BOOTP provides a means to notify a host of its assigned IP address, the IP address of a boot server host, and the name of a file to be loaded into memory and executed [1]. Other configuration information such as the local subnet mask, the local time offset, the addresses of default routers, and the addresses of various Internet servers can also be communicated to a host using BOOTP [2].

Unfortunately, the original BOOTP specification [1] left some issues of the protocol open to question. The exact behavior of BOOTP relay agents formerly called "BOOTP forwarding agents") was not clearly specified. Some parts of the overall protocol specification actually conflict, while other parts have been subject to misinterpretation, indicating that clarification is needed. This memo addresses these problems.

Since the introduction of BOOTP, the IEEE 802.5 Token Ring Network has been developed which presents a unique problem for BOOTP's particular message-transfer paradigm. This memo also suggests a solution for this problem.

NOTE: Unless otherwise specified in this document or a later document, the information and requirements specified througout this document also apply to extensions to BOOTP such as the Dynamic Host Configuration Protocol (DHCP) [3]. 


\subsection{Requirements}

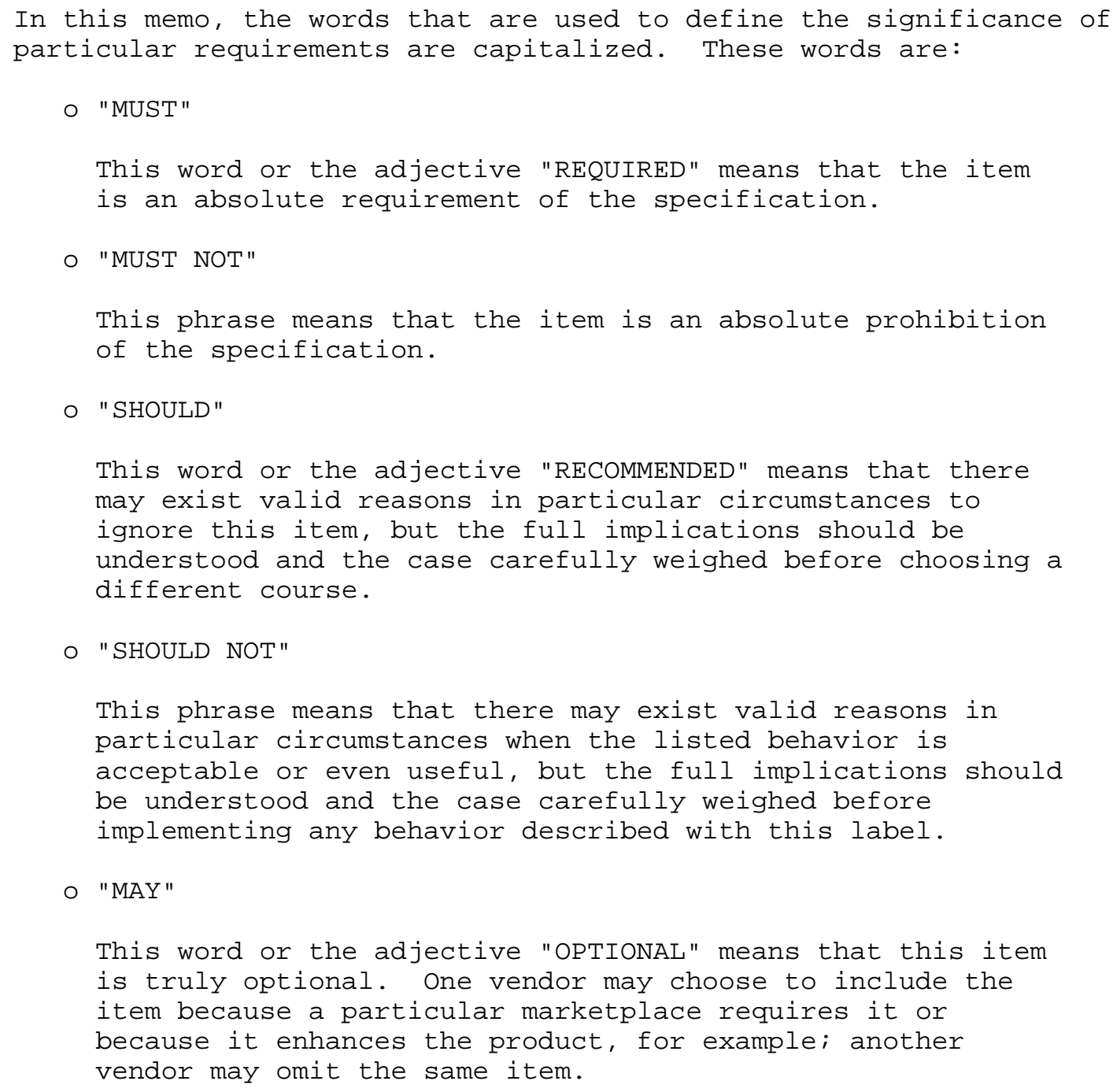




\section{BOOTREPLY}

A BOOTREPLY message is a BOOTP message sent from a BOOTP server to a BOOTP client, providing configuration information.

\section{Silently discard}

This memo specifies several cases where a BooTP entity is to "silently discard" a received BOOTP message. This means that the entity is to discard the message without further processing, and that the entity will not send any ICMP error message as a result. However, for diagnosis of problems, the entity SHOULD provide the capability of logging the error, including the contents of the silently-discarded message, and SHOULD record the event in a statistics counter.

\subsection{Data Transmission Order}

The order of transmission of the header and data described in this document is resolved to the octet level. Whenever a diagram shows a group of octets, the order of transmission of those octets is the normal order in which they are read in English. For example, in the following diagram, the octets are transmitted in the order they are numbered.

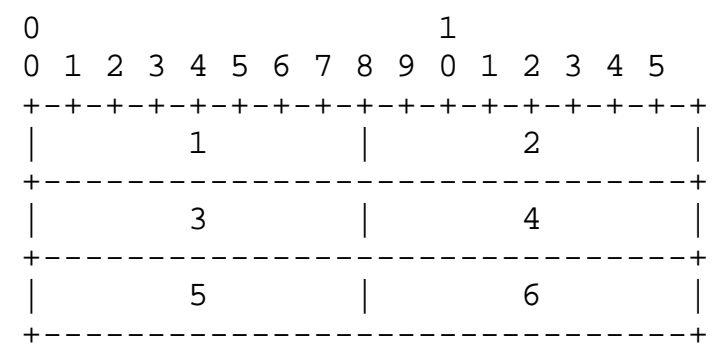

Whenever an octet represents a numeric quantity, the leftmost bit in the diagram is the high order or most significant bit. That is, the bit labeled 0 is the most significant bit. For example, the following diagram represents the value 170 (decimal).

$$
\begin{aligned}
& \begin{array}{llllllll}
0 & 1 & 2 & 3 & 4 & 5 & 6 & 7
\end{array} \\
& \text { +-+-+--+-+-+--+-+-+ } \\
& \left|\begin{array}{llllllll}
1 & 0 & 1 & 0 & 1 & 0 & 1 & 0
\end{array}\right| \\
& \text { +---------------+ }
\end{aligned}
$$

Similarly, whenever a multi-octet field represents a numeric quantity the leftmost bit of the whole field is the most significant bit. When a multi-octet quantity is transmitted the most significant octet 
is transmitted first.

2. General Issues

This section covers issues of general relevance to all BootP entities (clients, servers, and relay agents).

\subsection{General BOOTP Processing}

The following consistency checks SHOULD be performed on BOOTP messages:

- The IP Total Length and UDP Length must be large enough to contain the minimal BOOTP header of 300 octets (in the UDP data field) specified in [1].

NOTE: Future extensions to the BOOTP protocol may increase the size of BOOTP messages. Therefore, BOOTP messages which, according to the IP Total Length and UDP Length fields, are larger than the minimum size specified by [1] MUST also be accepted.

o The 'op' (opcode) field of the message must contain either the code for a BOOTREQUEST (1) or the code for a BOOTREPLY (2).

BOOTP messages not meeting these consistency checks MUST be silently discarded.

\subsection{Definition of the 'flags' Field}

The standard BOOTP message format defined in [1] includes a two-octet field located between the 'secs' field and the 'ciaddr' field. This field is merely designated as "unused" and its contents left unspecified, although section 7.1 of [1] does offer the following suggestion:

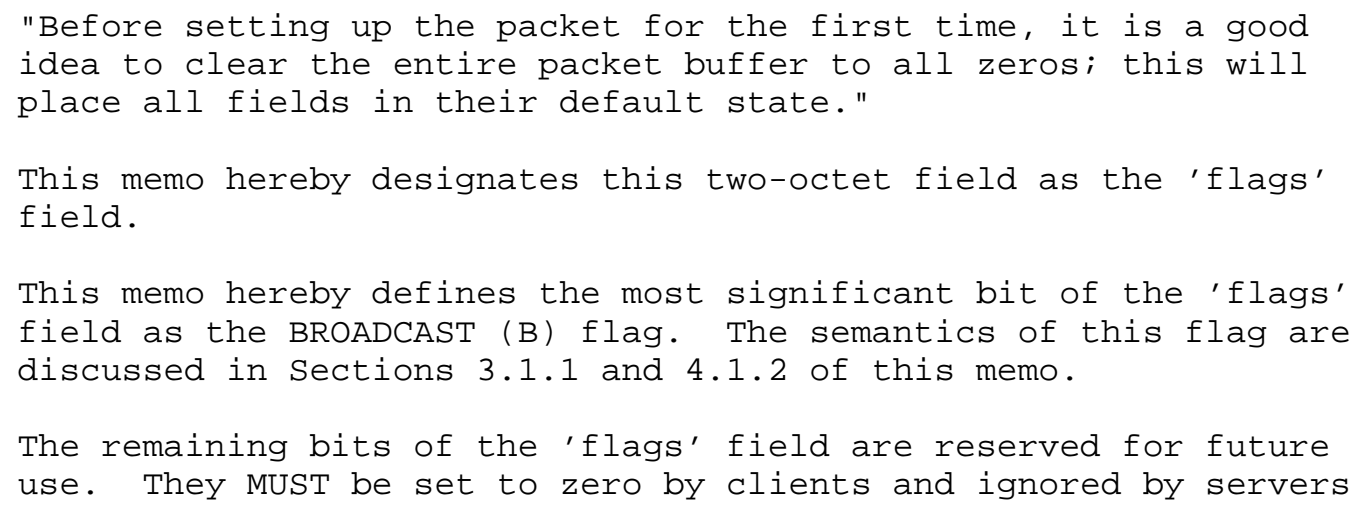


and relay agents.

The 'flags' field, then, appears as follows:

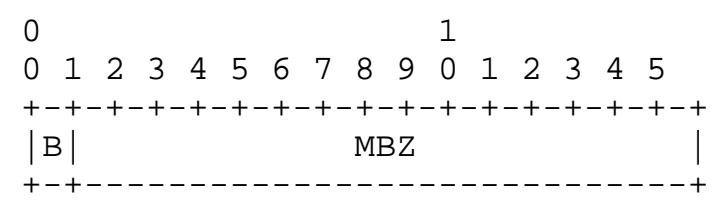

where:

B BROADCAST flag (discussed in Sections 3.1 .1 and 4.1.2)

MBZ MUST BE ZERO (reserved for future use)

The format of a BOOTP message is shown below. The numbers in parentheses indicate the size of each field in octets. 


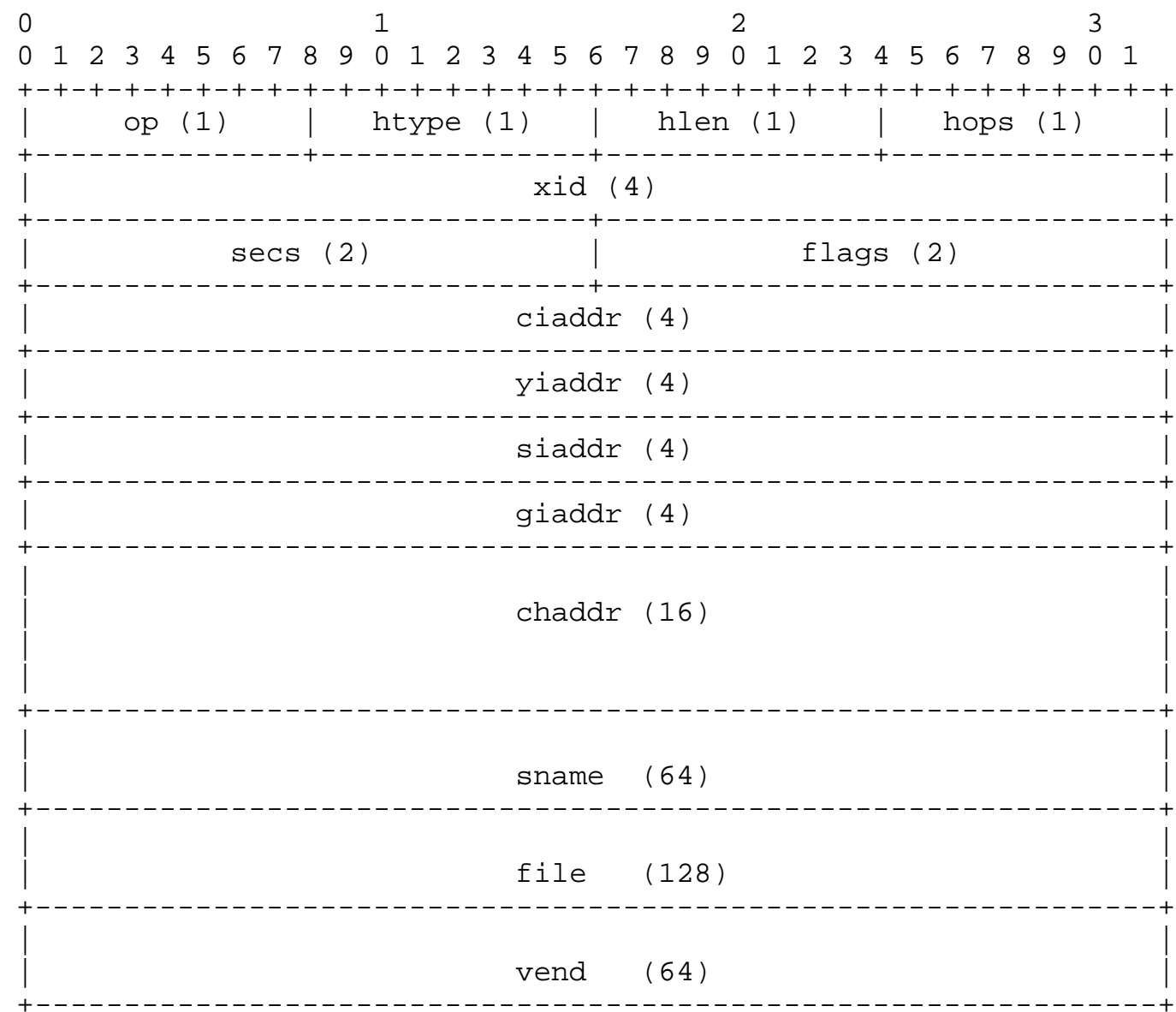

\subsection{Bit Ordering of Hardware Addresses}

The bit ordering used for link-level hardware addresses in the ' chaddr' field SHOULD be the same as the ordering used for the ARP protocol [4] on the client's link-level network (assuming ARP is defined for that network).

The 'Chaddr' field MUST be preserved as it was specified by the BOOTP client. A relay agent MUST NOT reverse the bit ordering of the 'chaddr' field even if it happens to be relaying a BOOTREQUEST between two networks which use different bit orderings.

\section{DISCUSSION :}

One of the primary reasons the 'chaddr' field exists is to enable BOOTP servers and relay agents to communicate directly 
with clients without the use of broadcasts. In practice, the contents of the 'chaddr' field is often used to create an ARPcache entry in exactly the same way the normal ARP protocol would have. Clearly, interoperability can only be achieved if a consistent interpretation of the 'chaddr' field is used.

As a practical example, this means that the bit ordering used for the 'chaddr' field by a BOOTP client on an IEEE 802.5 Token Ring network is the opposite of the bit ordering used by a BOOTP client on a DIX ethernet network.

\subsection{BOOTP Over IEEE 802.5 Token Ring Networks}

Special consideration of the client/server and client/relay agent interactions must be given to IEEE 802.5 networks because of nontransparent bridging.

The client SHOULD send its broadcast BOOTREQUEST with an All Routes Explorer RIF. This will enable servers/relay agents to cache the return route if they choose to do so. For those server/relay agents which cannot cache the return route (because they are stateless, for example), the BOOTREPLY message SHOULD be sent to the client's hardware address, as taken from the BOOTP message, with a spanning Tree Rooted RIF. The actual bridge route will be recorded by the client and server/relay agent by normal ARP processing code.

\section{DISCUSSION :}

In the simplest case, an unbridged, single ring network, the broadcast behavior of the BOOTP protocol is identical to that of Ethernet networks. However, a BOOTP client cannot know, a priori, that an 802.5 network is not bridged. In fact, the likelihood is that the server, or relay agent, will not know either.

Of the four possible scenerios, only two are interesting: where the assumption is that the 802.5 network is not bridged and it is, and the assumption that the network is bridged and it is not. In the former case, the Routing Information Field (RIF) will not be used; therefore, if the server/relay agent are on another segment of the ring, the client cannot reach it. In the latter case, the RIF field will be used, resulting in a few extraneous bytes on the ring. It is obvious that an almost immeasurable inefficiency is to be preferred over a complete failure to communicate. 
Given that the assumption is that RIF fields will be needed, it is necesary to determine the optimum method for the client to reach the server/relay agent, and the optimum method for the response to be returned.

3. BoOTP Client Behavior

This section clarifies various issues regarding BOOTP client behavior.

3.1 Client use of the 'flags' field

\subsubsection{The BROADCAST flag}

Normally, BOOTP servers and relay agents attempt to deliver BOOTREPLY messages directly to a client using unicast delivery. The IP destination address (in the IP header) is set to the BOOTP 'yiaddr' address and the link-layer destination address is set to the BOOTP 'chaddr' address. Unfortunately, some client implementations are unable to receive such unicast IP datagrams until they know their own IP address (thus we have a "chicken and egg" issue). Often, however, they can receive broadcast IP datagrams (those with a valid IP broadcast address as the IP destination and the link-layer broadcast address as the link-layer destination).

If a client falls into this category, it SHOULD set (to 1) the newly-defined BROADCAST flag in the 'flags' field of BOOTREPLY messages it generates. This will provide a hint to BOOTP servers and relay agents that they should attempt to broadcast their BOOTREPLY messages to the client.

If a client does not have this limitation (i.e., it is perfectly able to receive unicast BOOTREPLY messages), it SHOULD NOT set the BROADCAST flag (i.e., it SHOULD clear the BROADCAST flag to 0 ).

\section{DISCUSSION :}

This addition to the protocol is a workaround for old host implementations. Such implementations SHOULD be modified so that they may receive unicast BOOTREPLY messages, thus making use of this workaround unnecessary. In general, the use of this mechanism is discouraged.

\subsubsection{The remainder of the 'flags' field}

The remaining bits of the 'flags' field are reserved for future use. A client MUST set these bits to zero in all BOOTREQUEST messages it generates. A client MUST ignore these bits in all BOOTREPLY messages 
it receives.

3.2 Definition of the 'secs' field

The 'secs' field of a BOOTREQUEST message SHOULD represent the elapsed time, in seconds, since the client sent its first BOOTREQUEST message. Note that this implies that the 'secs' field of the first BOOTREQUEST message SHOULD be set to zero.

Clients SHOULD NOT set the 'secs' field to a value which is constant for all BOOTREQUEST messages.

\section{DISCUSSION :}

The original definition of the 'secs' field was vague. It was not clear whether it represented the time since the first BOOTREQUEST message was sent or some other time period such as the time since the client machine was powered-up. This has limited its usefulness as a policy control mechanism for BooTP servers and relay agents. Furthermore, certain client implementations have been known to simply set this field to a constant value or use incorrect byte-ordering. Incorrect byte-ordering usually makes it appear as if a client has been waiting much longer than it really has, so a relay agent will relay the BOOTREQUEST sooner than desired (usually immediately). These implementation errors have further undermined the usefulness of the 'secs' field. These incorrect implementations SHOULD be corrected.

\subsection{Use of the 'ciaddr' and 'yiaddr' fields}

If a BOOTP client does not know what IP address it should be using, the client SHOULD set the 'ciaddr' field to 0.0.0.0. If the client has the ability to remember the last IP address it was assigned, or it has been preconfigured with an IP address via some alternate mechanism, the client MAY fill the 'ciaddr' field with that IP address. If the client does place a non-zero IP address in the 'ciaddr' field, the client MUST be prepared to accept incoming unicast datagrams addressed to that IP address and also answer ARP requests for that IP address (if ARP is used on that network).

The BOOTP server is free to assign a different IP address (in the 'yiaddr' field) than the client expressed in 'ciaddr'. The client SHOULD adopt the IP address specified in 'yiaddr' and begin using it as soon as possible. 


\section{DISCUSSION :}

There are various interpretations about the purpose of the 'ciaddr' field and, unfortunately, no agreement on a single correct interpretation. One interpretation is that if a client is willing to accept whatever IP address the BOOTP server assigns to it, the client should always place 0.0.0.0 in the 'ciaddr' field, regardless of whether it knows its previouslyassigned address. Conversely, if the client wishes to assert that it must have a particular IP address (e.g., the IP address was hand-configured by the host administrator and BOOTP is only being used to obtain a boot file and/or information from the 'vend' field), the client will then fill the 'ciaddr' field with the desired IP address and ignore the IP address assigned by the BOOTP server as indicated in the 'yiaddr' field. An alternate interpretation holds that the client always fills the 'ciaddr' field with its most recently-assigned IP address (if known) even if that address may be incorrect. Such a client will still accept and use the address assigned by the BOOTP server as indicated in the 'yiaddr' field. The motivation for this interpretation is to aid the server in identifying the client and/or in delivering the BOOTREPLY to the client. Yet a third (mis)interpretation allows the client to use 'ciaddr' to express the client's desired IP address, even if the client has never used that address before or is not currently using that address.

The last interpretation is incorrect as it may prevent the BOOTREPLY from reaching the client. The server will usually unicast the reply to the address given in 'ciaddr' but the client may not be listening on that address yet, or the client may be connected to an incorrect subnet such that normal IP routing (correctly) routes the reply to a different subnet.

The second interpretation also suffers from the "incorrect subnet" problem.

The first interpretation seems to be the safest and most likely to promote interoperability.

\subsection{Interpretation of the 'giaddr' field}

The 'giaddr' field is rather poorly named. It exists to facilitate the transfer of BOOTREQUEST messages from a client, through BOOTP relay agents, to servers on different networks than the client. Similarly, it facilitates the delivery of BOOTREPLY messages from the servers, through BOOTP relay agents, back to the client. In no case does it represent a general IP router to be used by the client. A 
BOOTP client MUST set the 'giaddr' field to zero $(0.0 .0 .0)$ in all BOOTREQUEST messages it generates.

A BOOTP client MUST NOT interpret the 'giaddr' field of a BOOTREPLY message to be the IP address of an IP router. A BOOTP client SHOULD completely ignore the contents of the 'giaddr' field in BOOTREPLY messages.

\section{DISCUSSION :}

The semantics of the 'giaddr' field were poorly defined. Section 7.5 of [1] states:

"If 'giaddr' (gateway address) is nonzero, then the packets should be forwarded there first, in order to get to the server."

In that sentence, "get to" refers to communication from the client to the server subsequent to the BOOTP exchange, such as a TFTP session. Unfortunately, the 'giaddr' field may contain the address of a BOOTP relay agent that is not itself an IP router (according to [1], Section 8, fifth paragraph), in which case, it will be useless as a first-hop for TFTP packets sent to the server (since, by definition, non-routers don't forward datagrams at the IP layer).

Although now prohibited by section 4.1 .1 of this memo, the 'giaddr' field might contain a broadcast address according to section 8, sixth paragraph of [1]. Not only would such an address be useless as a router address, it might also cause the client to ARP for the broadcast address (since, if the client didn't receive a subnet mask in the BOOTREPLY message, it would be unable to recognize a subnet broadcast address). This is clearly undesirable.

To reach a non-local server, clients can obtain a first-hop router address from the "Gateway" subfield of the "Vendor Information Extensions" [2] (if present), or via the ICMP router discovery protocol [5] or other similar mechanism.

\subsection{Vendor information "magic cookie"}

It is RECOMMENDED that a BOOTP client always fill the first four octets of the 'vend' (vendor information) field of a BOOTREQUEST with a four-octet identifier called a "magic cookie." A BooTP client SHOULD do this even if it has no special information to communicate to the BOOTP server using the 'vend' field. This aids the BOOTP server in determining what vendor information format it should use in its BOOTREPLY messages. 
If a special vendor-specific magic cookie is not being used, a BOOTP client SHOULD use the dotted decimal value 99.130.83.99 as specified in [2]. In this case, if the client has no information to communicate to the server, the octet immediately following the magic cookie SHOULD be set to the "End" tag (255) and the remaining octets of the 'vend' field SHOULD be set to zero.

\section{DISCUSSION :}

Sometimes different operating systems or networking packages are run on the same machine at different times (or even at the same time!). Since the hardware address placed in the 'chaddr' field will likely be the same, BOOTREQUESTs from completely different BOOTP clients on the same machine will likely be difficult for a BOOTP server to differentiate. If the client includes a magic cookie in its BOOTREQUESTs, the server will at least know what format the client expects and can understand in corresponding BOOTREPLY messages.

\section{BOOTP Relay Agents}

In many cases, BOOTP clients and their associated BOOTP server(s) do not reside on the same IP network or subnet. In such cases, some kind of third-party agent is required to transfer BOOTP messages between clients and servers. Such an agent was originally referred to as a "BOOTP forwarding agent." However, in order to avoid confusion with the IP forwarding function of an IP router, the name "BOOTP relay agent" is hereby adopted instead.

\section{DISCUSSION :}

A BOOTP relay agent performs a task which is distinct from an IP router's normal IP forwarding function. While a router normally switches IP datagrams between networks more-or-less transparently, a BOOTP relay agent may more properly be thought to receive BOOTP messages as a final destination and then generate new BOOTP messages as a result. It is incorrect for a relay agent implementation to simply forward a BOOTP message "straight through like a regular packet."

This relay-agent functionality is most conveniently located in the routers which interconnect the clients and servers, but may alternatively be located in a host which is directly connected to the client subnet.

Any Internet host or router which provides BoOTP relay-agent capability MUST conform to the specifications in this memo. 


\subsection{General BOOTP Processing for Relay Agents}

All locally delivered UDP messages whose UDP destination port number is BOOTPS (67) are considered for special processing by the host or router's logical BOOTP relay agent.

In the case of a host, locally delivered datagrams are simply all datagrams normally received by that host, i.e., broadcast and multicast datagrams as well as unicast datagrams addressed to IP addresses of that host.

In the case of a router, locally delivered datagrams are broadcast and multicast datagrams as well as unicast datagrams addressed to IP addresses of that router. These are datagrams for which the router should be considered an end destination as opposed to an intermediate switching node. Thus a unicast datagram with an IP destination not matching any of the router's IP addresses is not considered for processing by the router's logical BOOTP relay agent.

Hosts and routers are usually required to silently discard incoming datagrams containing illegal IP source addresses. This is generally known as "Martian address filtering." One of these illegal addresses is 0.0.0.0 (or actually anything on network 0). However, hosts or routers which support a BOOTP relay agent MUST accept for local delivery to the relay agent BOOTREQUEST messages whose IP source address is 0.0.0.0. BOOTREQUEST messages from legal IP source addresses MUST also be accepted.

A relay agent MUST silently discard any received UDP messages whose UDP destination port number is BOOTPC (68).

\section{DISCUSSION :}

There should be no need for a relay agent to process messages addressed to the BOOTPC port. Careful reading of the original BOOTP specification [1] will show this. Nevertheless, some relay agent implementations incorrectly relay such messages.

The consistency checks specified in Section 2.1 SHOULD be performed by the relay agent. BOOTP messages not meeting these consistency checks MUST be silently discarded.

\subsubsection{BOOTREQUEST Messages}

Some configuration mechanism MUST exist to enable or disable the relaying of BOOTREQUEST messages. Relaying MUST be disabled by default. 
When the BOOTP relay agent receives a BOOTREQUEST message, it MAY use the value of the 'secs' (seconds since client began booting) field of the request as a factor in deciding whether to relay the request. If such a policy mechanism is implemented, its threshold sHoULD be configurable.

\section{DISCUSSION :}

To date, this feature of the BOOTP protocol has not necessarily been shown to be useful. See section 3.2 for a discussion.

The relay agent MUST silently discard BOOTREQUEST messages whose 'hops' field exceeds the value 16. A configuration option SHOULD be provided to set this threshold to a smaller value if desired by the network manager. The default setting for a configurable threshold SHOULD be 4 .

If the relay agent does decide to relay the request, it MUST examine the 'giaddr' ("gateway" IP address) field. If this field is zero, the relay agent MUST fill this field with the IP address of the interface on which the request was received. If the interface has more than one IP address logically associated with it, the relay agent SHOULD choose one IP address associated with that interface and use it consistently for all Bootp messages it relays. If the 'giaddr' field contains some non-zero value, the 'giaddr' field MUST NOT be modified. The relay agent MUST NOT, under any circumstances, fill the 'giaddr' field with a broadcast address as is suggested in [1] (Section 8, sixth paragraph).

The value of the 'hops' field MUST be incremented.

All other BOOTP fields MUST be preserved intact.

At this point, the request is relayed to its new destination (or destinations). This destination MUST be configurable. Further, this destination configuration SHOULD be independent of the destination configuration for any other so-called "broadcast forwarders" (e.g., for the UDP-based TFTP, DNS, Time, etc. protocols).

\section{DISCUSSION :}

The network manager may wish the relaying destination to be an IP unicast, multicast, broadcast, or some combination. A configurable list of destination IP addresses provides good flexibility. More flexible configuration schemes are encouraged. For example, it may be desirable to send to the limited broadcast address $(255.255 .255 .255)$ on specific physical interfaces. However, if the BOOTREQUEST message was 
received as a broadcast, the relay agent MUST NOT rebroadcast the BOOTREQUEST on the physical interface from whence it came.

A relay agent MUST use the same destination (or set of destinations) for all BOOTREQUEST messages it relays from a given client.

\section{DISCUSSION :}

At least one known relay agent implementation uses a roundrobin scheme to provide load balancing across multiple BOOTP servers. Each time it receives a new BOOTREQUEST message, it relays the message to the next BOOTP server in a list of servers. Thus, with this relay agent, multiple consecutive BOOTREQUEST messages from a given client will be delivered to different servers.

Unfortunately, this well-intentioned scheme reacts badly with DHCP [3] and perhaps other variations of the BOOTP protocol which depend on multiple exchanges of BOOTREQUEST and BOOTREPLY messages between clients and servers. Therefore, all BOOTREQUEST messages from a given client MUST be relayed to the same destination (or set of destinations).

One way to meet this requirement while providing some loadbalancing benefit is to hash the client's link-layer address (or some other reliable client-identifying information) and use the resulting hash value to select the appropriate relay destination (or set of destinations). The simplest solution, of course, is to not use a load-balancing scheme and just relay ALL received BOOTREQUEST messages to the same destination (or set of destinations).

When transmitting the request to its next destination, the relay agent may set the IP Time-To-Live field to either the default value for new datagrams originated by the relay agent, or to the TTL of the original BOOTREQUEST decremented by (at least) one.

\section{DISCUSSION :}

As an extra precaution against BOOTREQUEST loops, it is preferable to use the decremented TTL from the original BOOTREQUEST. Unfortunately, this may be difficult to do in some implementations.

If the BOOTREQUEST has a UDP checksum (i.e., the UDP checksum is non-zero), the checksum must be recalculated before 
transmitting the request.

\subsubsection{BOOTREPLY Messages}

BOOTP relay agents relay BOOTREPLY messages only to BOOTP clients. It is the responsibility of BOOTP servers to send BOOTREPLY messages directly to the relay agent identified in the 'giaddr' field. Therefore, a relay agent may assume that all BOOTREPLY messages it receives are intended for BOOTP clients on its directly-connected networks.

When a relay agent receives a BOOTREPLY message, it should examine the BOOTP 'giaddr', 'yiaddr', 'chaddr', 'htype', and 'hlen' fields. These fields should provide adequate information for the relay agent to deliver the BOOTREPLY message to the client.

The 'giaddr' field can be used to identify the logical interface from which the reply must be sent (i.e., the host or router interface connected to the same network as the BOOTP client). If the content of the 'giaddr' field does not match one of the relay agent's directly-connected logical interfaces, the BOOTREPLY messsage MUST be silently discarded.

The 'htype', 'hlen', and 'chaddr' fields supply the link-layer hardware type, hardware address length, and hardware address of the client as defined in the ARP protocol [4] and the Assigned Numbers document [6]. The 'yiaddr' field is the IP address of the client, as assigned by the BoOTP server.

The relay agent SHOULD examine the newly-defined BROADCAST flag (see Sections 2.2 and 3.1 .1 for more information). If this flag is set to 1, the reply SHOULD be sent as an IP broadcast using the IP limited broadcast address 255.255.255.255 as the IP destination address and the link-layer broadcast address as the link-layer destination address. If the BROADCAST flag is cleared (0), the reply SHOULD be sent as an IP unicast to the IP address specified by the 'yiaddr' field and the link-layer address specified in the 'chaddr' field. If unicasting is not possible, the reply MAY be sent as a broadcast, in which case it SHOULD be sent to the link-layer broadcast address using the IP limited broadcast address 255.255.255.255 as the IP destination address.

\section{DISCUSSION :}

The addition of the BROADCAST flag to the protocol is a workaround to help promote interoperability with certain client implementations. 
Note that since the 'flags' field was previously defined in [1] simply as an "unused" field, it is possible that old client or server implementations may accidentally and unknowingly set the new BROADCAST flag. It is actually expected that such implementations will be rare (most implementations seem to zero-out this field), but interactions with such implementations must nevertheless be considered. If an old client or server does set the BROADCAST flag to 1 incorrectly, conforming relay agents will generate broadcast BOOTREPLY messages to the corresponding client. The BOOTREPLY messages should still properly reach the client, at the cost of one (otherwise unnecessary) additional broadcast. This, however, is no worse than a server or relay agent which always broadcasts its BOOTREPLY messages.

Older client or server implementations which accidentally set the BROADCAST flag SHOULD be corrected to properly comply with this newer specification.

All BOOTP fields MUST be preserved intact. The relay agent MUST NOT modify any BOOTP field of the BOOTREPLY message when relaying it to the client.

The reply MUST have its UDP destination port set to BOOTPC (68).

If the BOOTREPLY has a UDP checksum (i.e., the UDP checksum is non-zero), the checksum must be recalculated before transmitting the reply.

5. BOOTP Server Behavior

This section provides clarifications on the behavior of BOOTP servers.

\subsection{Reception of BOOTREQUEST Messages}

All received UDP messages whose UDP destination port number is BOOTPS (67) are considered for processing by the BOOTP server.

Hosts and routers are usually required to silently discard incoming datagrams containing illegal IP source addresses. This is generally known as "Martian address filtering." One of these illegal addresses is 0.0.0.0 (or actually anything on network 0). However, hosts or routers which support a BOOTP server MUST accept for local delivery to the server BOOTREQUEST messages whose IP source address is 0.0.0.0. BOOTREQUEST messages from legal IP source addresses MUST also be accepted. 
A BOOTP server MUST silently discard any received UDP messages whose UDP destination port number is BOOTPC (68).

DISCUSSION :

There should be no need for a BOOTP server to process messages addressed to the BOOTPC port. Careful reading of the original BOOTP specification [1] will show this.

The consistency checks specified in Section 2.1 SHould be performed by the BOOTP server. BOOTP messages not meeting these consistency checks MUST be silently discarded.

5.2 Use of the 'secs' field

When the BOOTP server receives a BOOTREQUEST message, it MAY use the value of the 'secs' (seconds since client began booting) field of the request as a factor in deciding whether and/or how to reply to the request.

\section{DISCUSSION :}

To date, this feature of the BOoTP protocol has not necessarily been shown to be useful. See Section 3.2 for a discussion.

\subsection{Use of the 'ciaddr' field}

There have been various client interpretations of the 'ciaddr' field for which section 3.3 should be consulted. A BOOTP server SHOULD be prepared to deal with these varying interpretations. In general, the 'ciaddr' field SHOULD NOT be trusted as a sole key in identifying a client; the contents of the 'ciaddr', 'chaddr', 'htype', and 'hlen' fields, and probably other information (perhaps in the 'file' and 'vend' fields) SHOULD all be considered together in deciding how to respond to a given client.

BOOTP servers SHOULD preserve the contents of the 'ciaddr' field in BOOTREPLY messages; the contents of 'ciaddr' in a BOOTREPLY message SHOULD exactly match the contents of 'ciaddr' in the corresponding BOOTREQUEST message.

\section{DISCUSSION :}

It has been suggested that a client may wish to use the contents of 'ciaddr' to further verify that a particular BOOTREPLY message was indeed intended for it. 


\subsection{Strategy for Delivery of BOOTREPLY Messages}

Once the BOOTP server has created an appropriate BOOTREPLY message, that BOOTREPLY message must be properly delivered to the client.

The server SHOULD first check the 'ciaddr' field. If the 'ciaddr' field is non-zero, the BOOTREPLY message SHOULD be sent as an IP unicast to the IP address identified in the 'ciaddr' field. The UDP destination port MUST be set to BOOTPC (68). However, the server MUST be aware of the problems identified in section 3.3. The server MAY choose to ignore the 'ciaddr' field and act as if the 'ciaddr' field contains 0.0 .0 .0 (and thus continue with the rest of the delivery algorithm below).

The server SHOULD next check the 'giaddr' field. If this field is non-zero, the server SHOULD send the BOOTREPLY as an IP unicast to the IP address identified in the 'giaddr' field. The UDP destination port MUST be set to BOOTPS (67). This action will deliver the BOOTREPLY message directly to the BOOTP relay agent closest to the client; the relay agent will then perform the final delivery to the client. If the BOOTP server has prior knowledge that a particular client cannot receive unicast BOOTREPLY messages (e.g., the network manager has explicitly configured the server with such knowledge), the server MAY set the newly-defined BROADCAST flag to indicate that relay agents SHOULD broadcast the BOOTREPLY message to the client. Otherwise, the server MUST preserve the state of the BROADCAST flag so that the relay agent can correctly act upon it.

If the 'giaddr' field is set to 0.0.0.0, then the client resides on one of the same networks as the BOOTP server. The server SHOULD examine the newly-defined BROADCAST flag (see Sections 2.2, 3.1.1 and 4.1.2 for more information). If this flag is set to 1 or the server has prior knowledge that the client is unable to receive unicast BOOTREPLY messages, the reply SHOULD be sent as an IP broadcast using the IP limited broadcast address 255.255.255.255 as the IP destination address and the link-layer broadcast address as the link-layer destination address. If the BROADCAST flag is cleared (0), the reply SHOULD be sent as an IP unicast to the IP address specified by the 'yiaddr' field and the link-layer address specified in the 'chaddr' field. If unicasting is not possible, the reply MAY be sent as a broadcast in which case it SHOULD be sent to the linklayer broadcast address using the IP limited broadcast address 255.255.255.255 as the IP destination address. In any case, the UDP destination port MUST be set to BOOTPC (68). 


\section{DISCUSSION :}

The addition of the BROADCAST flag to the protocol is a workaround to help promote interoperability with certain client implementations.

The following table summarizes server delivery decisions for BOOTREPLY messages based upon information in BOOTREQUEST messages:

\begin{tabular}{|c|c|c|c|c|c|}
\hline BOOTREQ & ST field & & BOOTREPLY & -ues for UDP, IP, & link-layer \\
\hline ' ciaddr' & 'giaddr' & B & UDP dest & IP destination & link dest \\
\hline non-zero & $\mathrm{x}$ & $\mathrm{x}$ & BOOTPC (68) & 'ciaddr' & normal \\
\hline 0.0 .0 .0 & non-zero & $\mathrm{X}$ & BOOTPS (67) & 'giaddr' & normal \\
\hline 0.0 .0 .0 & 0.0 .0 .0 & 0 & BOOTPC (68) & 'yiaddr' & 'chaddr' \\
\hline 0.0 .0 .0 & 0.0 .0 .0 & 1 & BООTРC (68) & 255.255 .255 .255 & broadcast \\
\hline
\end{tabular}

$$
\begin{aligned}
\mathrm{B}= & \text { BROADCAST flag } \\
\mathrm{X}= & \text { Don't care } \\
\text { normal = } & \text { determine from the given IP destination using normal } \\
& \text { IP routing mechanisms and/or ARP as for any other } \\
& \text { normal datagram }
\end{aligned}
$$

Acknowledgements

The author would like to thank Gary Malkin for his contribution of the "BOOTP over IEEE 802.5 Token Ring Networks" section, and Steve Deering for his observations on the problems associated with the 'giaddr' field.

Ralph Droms and the many members of the IETF Dynamic Host Configuration and Router Requirements working groups provided ideas for this memo as well as encouragement to write it.

Philip Almquist and David Piscitello offered many helpful suggestions for improving the clarity, accuracy, and organization of this memo. These contributions are graciously acknowledged. 


\section{References}

[1] Croft, B., and J. Gilmore, "Bootstrap Protocol (BOOTP)", RFC 951, Stanford University and Sun Microsystems, september 1985.

[2] Reynolds, J., "BOOTP Vendor Information Extensions", RFC 1497, USC/Information Sciences Institute, August 1993. This RFC is occasionally reissued with a new number. Please be sure to consult the latest version.

[3] Droms, R., "Dynamic Host Configuration Protocol", RFC 1541, Bucknell University, October 1993.

[4] Plummer, D., "An Ethernet Address Resolution Protocol", STD 37 , RFC 826, MIT, November 1982 .

[5] Deering, S., "ICMP Router Discovery Messages", RFC 1256, Xerox PARC, September 1991 .

[6] Reynolds, J., and J. Postel, "Assigned Numbers", STD 2, RFC 1340, USC/Information Sciences Institute, July, 1992. This RFC is periodically reissued with a new number. Please be sure to consult the latest version. 


\section{Security Considerations}

There are many factors which make BOOTP in its current form quite insecure. BOOTP is built directly upon UDP and IP which are as yet inherently insecure themselves. Furthermore, BOOTP is generally intended to make maintenance of remote and/or diskless hosts easier. While perhaps not impossible, configuring such hosts with passwords or keys may be difficult and inconvenient. This makes it difficult to provide any form of reasonable authentication between servers and clients.

Unauthorized BOOTP servers may easily be set up. Such servers can then send false and potentially disruptive information to clients such as incorrect or duplicate IP addresses, incorrect routing information (including spoof routers, etc.), incorrect domain nameserver addresses (such as spoof nameservers), and so on. Clearly, once this "seed" mis-information is planted, an attacker can further compromise the affected systems.

Unauthorized BOOTP relay agents may present some of the same problems as unauthorized BOOTP servers.

Malicious BOOTP clients could masquerade as legitimate clients and retrieve information intended for those legitimate clients. Where dynamic allocation of resources is used, a malicious client could claim all resources for itself, thereby denying resources to legitimate clients.

Author's Address

Walt Wimer

Network Development

Carnegie Mellon University

5000 Forbes Avenue

Pittsburgh, PA 15213-3890

Phone: (412) 268-6252

EMail: Walter.Wimer@CMU.EDU 